\title{
Highly Sensitive Nitric Oxide Detection Using X-ray Photoelectron Spectroscopy
}

\author{
Manish Dubey, Steven L. Bernasek and Jeffrey Schwartz* \\ Department of Chemistry, Princeton University, Princeton, NJ 08544-1009
}

\section{Supporting Information}

Detailed descriptions of monolayer preparation and XPS analysis; IR spectrum of pure hematin and hematin diacyl chloride, $\mathbf{3}$; XPS survey scan of $\mathbf{4}$, showing N1s and Fe2p peaks; and a table showing the area under the N1s peaks of $\mathbf{4}$ and $\mathbf{5}$ are given. 
1. Monolayer preparation. A wafer of single-crystal $\mathrm{Si}(100)$ was cut into $1 \mathrm{~cm} \mathrm{X} 1 \mathrm{~cm}$ coupons and cleaned in different organic solvents. The clean silicon coupons [Si-1] were etched with aqueous $\mathrm{HF}$ to remove existing oxide and were reoxidized in fuming $\mathrm{HNO}_{3}$ for $1 \mathrm{~min}$, followed by intensive rinsing in Milli-Q water. Phosphonic acid 1 selfassembles on the cleaned coupons using the T-BAG method in which the coupons are held vertically in a $25 \mu \mathrm{M}$ solution of the acid in dry THF. The solvent was allowed to evaporate at room temperature. The coated coupons were then heated in an oven at $140^{\circ} \mathrm{C}$ for $48 \mathrm{~h}$, after which time they were cleaned and characterized to obtain a monolayer of the phosphonate 2 .

2. XPS Analysis. All samples were analyzed by XPS using a Phoibos 150 hemispherical energy analyzer (SPECS) and a monochromatic Al source $(1486.6 \mathrm{eV})$. The spectrometer was calibrated to the position of the $3 \mathrm{~d}_{5 / 2}$ line of sputter-cleaned Ag at a binding energy of $368.25 \mathrm{eV}$ and the $\mathrm{C} 1 \mathrm{~s}$ line of highly ordered pyrolytic graphite (HOPG) at $284.5 \mathrm{eV}$. A pass energy of $100 \mathrm{eV}$ was used for wide range scans, while a $10 \mathrm{eV}$ pass energy were used for high-resolution measurements. All samples were attached to the sample holder by a stainless steel pin to ensure good electrical contact. Curve fitting of the core XPS lines was carried out using CasaXPS software using a Gaussian-Lorentzian product function and a nonlinear Shirley background subtraction. 
Supporting figure 1: IR spectrum of (a) pure hematin and (b) hematin diacyl chloride, 3; note the appearance of a new peak at $1810 \mathrm{~cm}^{-1}$ for acid chloride.

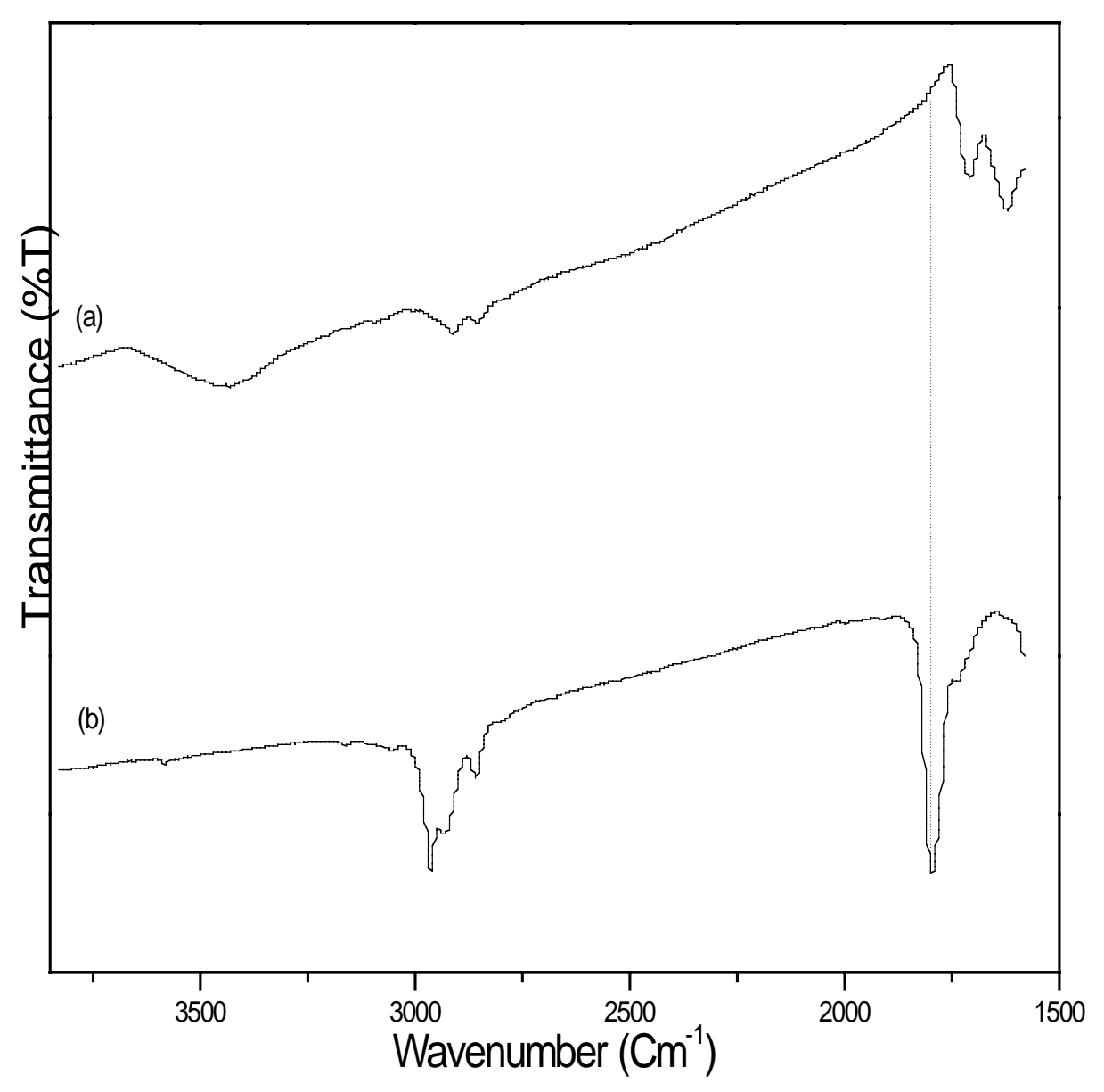


Supporting figure 2: XPS survey scan of 4; N1s and Fe2p peaks confirm the presence of hematin on the surface.

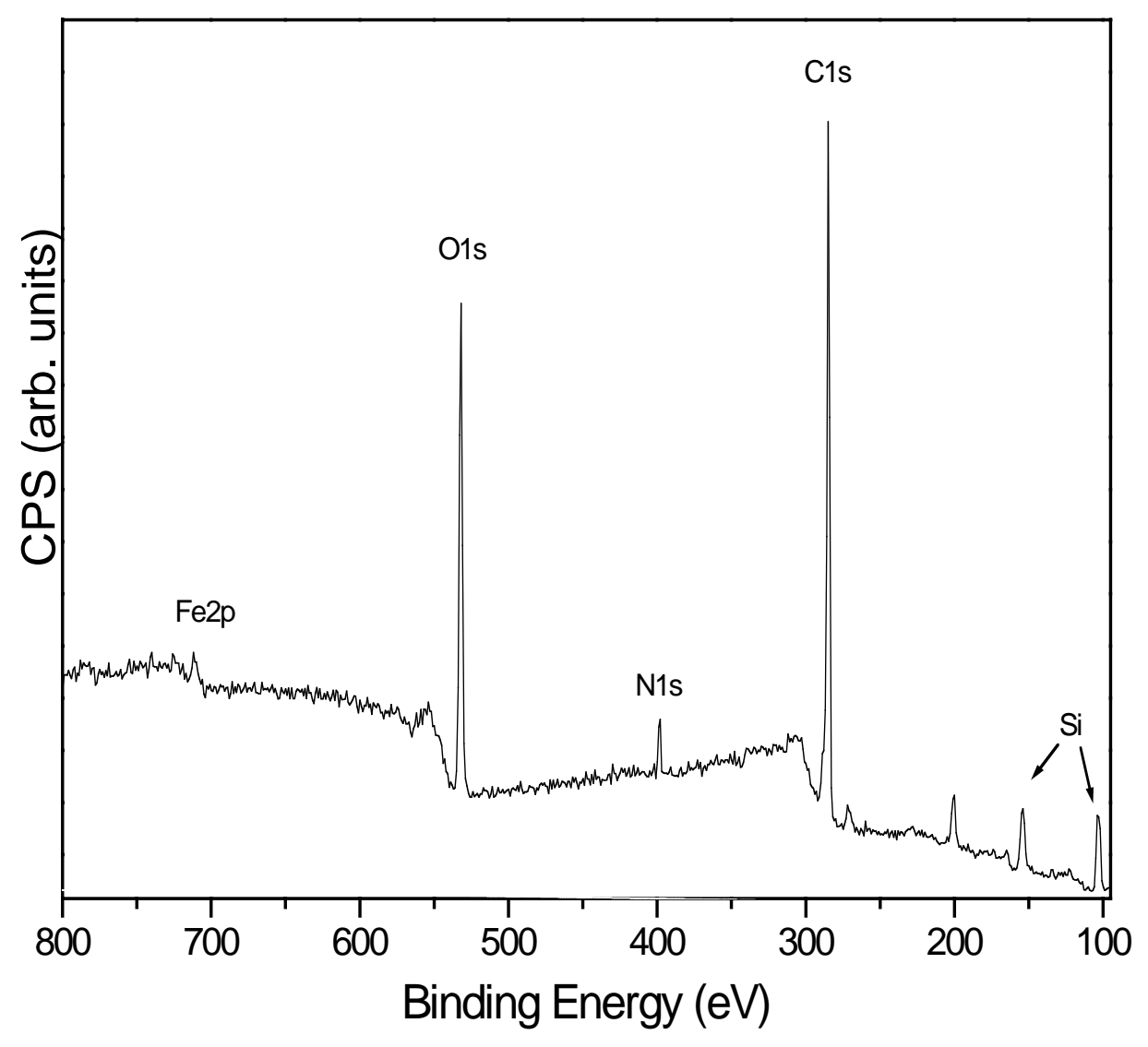


Supporting table 1: The areas under the N1s peaks of $\mathbf{4}$ and $\mathbf{5}$.

\begin{tabular}{|c|c|c|c|}
\hline & N1 & N2 & N3 \\
\hline $\mathbf{4}$ & --- & 1007.3 & --- \\
\hline $\mathbf{5}$ & 808.7 & 215.2 & 210.9 \\
\hline
\end{tabular}

